NEUROGASTROENTEROLOGY

\title{
CD55 expression patterns on intestinal neuronal tissue are divergent from the brain
}

\author{
K A Gelderman, H J M A A Zijlmans, M J Vonk, A Gorter
}

Gut 2004;53:507-513. doi: 10.1136/gut.2003.026773

See end of article for authors' affiliations ....................

Correspondence to: Dr A Gorter, Department of Pathology, Leiden University Medical Centre, Building 1, L1-Q, P O Box 9600,2300 RC Leiden, the Netherlands;

A.Gorter@lumc.nl

Accepted for publication 28 October 2003
Background and objectives: In this study, we investigated how enteric plexuses protect themselves from complement mediated attack. For this purpose, the expression patterns of membrane bound complement regulatory proteins ( $\mathrm{mCRP}$ ) and their association with C3 deposition was determined. In addition, $\mathrm{mCRP}$ expression patterns of enteric plexuses were compared with those in the central nervous system (CNS). Methods: Immunohistochemical stainings were performed to discriminate neuronal cells from glial cells and to detect the presence of CD46, CD55, CD59, and C3d. RNA in situ hybridisation (RISH) was used to determine the cell types that produce CD55 mRNA.

Results: Enteric plexuses minimally expressed CD46 whereas CD55 and CD59 were highly expressed. CD55 expression was also observed in a ring around Auerbach's plexuses which was not observed for CD46 and CD59. C3d was deposited around the plexuses but plexus cells themselves did not stain for C3d. In contrast with CNS neurones, enteric neurones were shown to express CD55 whereas enteric glial cells did not. This was confirmed with CD55 RISH. Phospholipase C mediated cleavage of CD55 demonstrated that CD55 was most likely attached to elastic fibres surrounding the plexus. Attached CD55 might protect CD55 negative glial cells from complement mediated injury during inflammatory reactions. CD55 on elastic fibres surrounding the plexuses most likely originated from enteric neuronal cells.

Conclusion: In contrast with the CNS, enteric neurones express CD55 and enteric glial cells lack CD55 expression. CD55, produced by neuronal cells, attached to elastic fibres surrounding the plexuses is proposed to protect the CD55 negative glial cells within plexuses.
$\mathrm{T}$ he enteric nervous system is responsible for regulation of gastrointestinal motility and fluid movement. ${ }^{12}$ Nerve plexuses are found at two levels throughout the gastrointestinal tract: the myenteric plexus or Auerbach's plexus is situated between the circular and longitudinal muscle layers and the submucosal plexuses (Henle's plexus and Meissner's plexus) are located in the submucosa. ${ }^{3}$ Enteric plexuses consist of two cell types: neuronal cells and enteric glial cells. Neuronal cell bodies are organised in plexuses that are interconnected by nerve fibre bundles. Enteric glial cells are, in contrast with Schwann cells, not able to form basal laminae around axons. Therefore, they form groups to protect neuronal cells. Enteric glial cells have similar properties as astrocytes in the brain. ${ }^{4}$

In the gut, where inflammation is a common phenomenon, plexuses need sufficient protection against complement activation. For several intestinal diseases, abnormalities of these plexuses are thought to play a role in pathogenesis. Previously, associations have been reported between plexus abnormalities and inflammatory conditions, ${ }^{56}$ for example in Crohn's disease ${ }^{5}$ and in Chagas' disease. ${ }^{8}$ Some studies describe a role for complement in these pathological processes, especially for the lectin pathway. ${ }^{9}{ }^{10}$ Abnormalities in expression of membrane bound complement regulatory proteins (mCRP) on plexus cells might affect the course of these diseases by allowing altered complement activation that might influence peripheral neuronal function. Decreased expression of mCRP may lead to tissue injury whereas overexpression of mCRP, as described for different tumour types, ${ }^{11}$ might be beneficial for tumour cells originating from the enteric plexuses.

To protect themselves against complement mediated attack, most cells express one or more mCRP. On cell surfaces, the complement cascade is regulated at two main levels. At the C3 level, complement receptor type l (CD35),
CD46 (membrane cofactor protein), and CD55 (decay accelerating factor) regulate the activity of the complement C3 and C5 convertases. ${ }^{12}{ }^{13}$ At the level of C9, formation of the membrane attack complex of complement on the cell membrane is restricted by CD59. ${ }^{14}$ Previously, expression levels of CD46, CD55, and CD59 on different cells in the central nervous system (CNS) have been studied. It was found that microglia in the CNS express CD55 and CD59 abundantly and CD46 in smaller amounts. Astrocytes weakly expressed all three mCRP. Neurones however were negative for CD55 and expressed CD46 and CD59 only in minimal amounts. ${ }^{15-17}$ Due to this low expression of mCRP, neurones are particularly susceptible to complement mediated injury ${ }^{17}$ which may be disadvantageous in the gut. In the present study, we have investigated mCRP expression patterns on enteric plexuses to determine how enteric plexus neurones are protected from complement mediated attack. In addition, we have compared mCRP expression patterns of enteric plexuses with mCRP expression patterns in the CNS.

\section{MATERIALS AND METHODS}

\section{Tissue material}

Tissue specimens of surgically removed colorectal cancer tissue were collected at the Department of Pathology of the LUMC (Leiden, the Netherlands). Tissue specimens were frozen in $-80^{\circ} \mathrm{C}$ isopentane or processed to be embedded in paraffin wax. Normal colon tissue originated from colorectal

Abbreviations: CNS, central nervous system; mCRP, membrane bound complement regulatory proteins; PIPLC, phosphatidylinositol specific phospholipase C; RISH, RNA in situ hybridisation; mAb, monoclonal antibodies; PGP9.5, protein gene product 9.5; PBS, phosphate buffered saline; PCR, polymerase chain reaction 
cancer patients and consisted of tumour free pieces of colon. Colorectal tissue of 43 patients with colorectal cancer (including eight normal colon tissue sections) that contained plexuses were used in this study. Brain tissue (cerebrum) of 10 persons was obtained from autopsies, frozen in isopentane and stored at $-80^{\circ} \mathrm{C}$. Approval for use was according to the medical ethics committee of the LUMC.

\section{Antibodies}

The following primary monoclonal antibodies (mAb) were used: Bric216 (anti-CD55, IgGl; IBGRL, Bristol, UK), MBCl (anti-CD55, IgGl; a kind gift from Professor BP Morgan, Cardiff, UK), J4-48 (anti-CD46, IgGl; CLB Sanquin, Amsterdam, the Netherlands), Bric 229 (anti-CD59, IgG2b; IBGRL), NCL-S100p (rabbit-antihuman S100 protein; Novocastra, Newcastle upon Tyne, UK), protein gene product 9.5 (PGP9.5, rabbit Ig; Biogenesis Ltd, Poole, UK), mouseantihuman elastin (IgGl; Novocastra), and mouse-antihuman C3d (Quidel Corporation, San Diego, California, USA). Secondary mAb used for immunohistochemistry were goat-antirabbit Ig-HRP (Dako, Glostrup, Denmark) and rabbit-antimouse Ig PowerVision+ (Dako).

\section{Immunohistochemistry}

Cryostat sections of $5 \mu \mathrm{m}$ were dried $\left(60^{\circ} \mathrm{C}\right.$, overnight $)$ and fixed in acetone ( 10 minutes). Sections were stained as described previously. ${ }^{18}$ The colon carcinoma tissue series and normal colon tissue series were simultaneously stained in one experiment to allow comparison. The intensity of staining of the plexuses was scored as negative (0), weak (1), moderate (2), or strong (3), referring to the most intensely stained part of all sections for a certain marker (3). The percentage of positive cells within a plexus was scored as sporadic ( 1 ; $1-5 \%)$, local $(2 ; 6-25 \%)$, occasional $(3 ; 26-50 \%)$, majority (4; $51-75 \%)$, or large majority $(5 ; 76-100 \%)$, according to the method described by Ruiter and colleagues. ${ }^{19}$ Individual scores for intensity and percentage of positive cells were added for each section to obtain a single outcome. These cumulative scores were analysed using the Mann-Whitney test.

\section{Phosphatidylinositol specific phospholipase C mediated cleavage of CD55}

Cryostat sections of $5 \mu \mathrm{m}$ were dried $\left(60^{\circ} \mathrm{C}\right.$, overnight). Sections were washed twice with phosphate buffered saline (PBS, five minutes) and subsequently treated with $0.1 \mathrm{U} / \mathrm{ml}$ phosphatidylinositol specific phospholipase C (PIPLC), diluted in PBS for 30 minutes at $37^{\circ} \mathrm{C}$. Then, sections were fixed in acetone for 10 minutes. After washing three times with PBS, CD55 staining was performed as described under the immunohistochemistry section above.

\section{Immunofluorescence}

The same protocol was used as described for cryostat sections (see immunohistochemistry section). Primary antibodies however were detected with Alexa-546 conjugated goatantimouse IgG (Molecular Probes, Leiden, the Netherlands) and/or FITC conjugated goat-antirabbit-Ig (Nordic Immunologicals, Tilburg, the Netherlands). Secondary Abs were diluted in PBS containing $1 \%$ bovine serum albumin and incubated for 30 minutes in the dark. Double stained sections were mounted in Mowiol 40-88 (Aldrich Chemie, Steinheim, Germany) and examined using a LSM 510 confocal microscope (Zeiss, Jena, Germany).

\section{Cloning of CD55 and $\beta$-actin CDNA}

cDNA was prepared from frozen human spleen. By using two oligonucleotide primers, chosen on the basis of a known sequence of human CD55 and $\beta$-actin (EMBL Genbank databases, accession Nos BC001288.1 and XM037235.1), cDNA encoding for CD55 and $\beta$-actin was amplified. Primer sequences were CGT TGC CAG AGT GCA GAG AAA (forward) and CCA AGC AAA CCT GTC AAC GTG (reverse), resulting in a 469 bp fragment for CD55. Primer sequences for $\beta$-actin were TCT GGC ACC ACA CCT TCT ACA A (forward) and GTG GTG GTG AAG CTG TAG CCG (reverse). Polymerase chain reaction (PCR) products were cloned into pGEM-3Zf(+) vectors (Promega, Madison, Wisconsin, USA) after linearising the vector with SmaI. The vectors were transferred to Escherichia coli strain Top 10 (Invitrogen Corp., San Diego, California, USA). Plasmids were isolated by using the QIAfilter Maxi KITS protocol (Qiagen GmbH, Hilden, Germany) and the sequence of the PCR products was confirmed by DNA sequencing.

\section{Preparation of $\beta$-actin and CD55 RNA probes}

Recombinant plasmids were linearised by cutting with either EcoR I or BamH I (both Boehringer, Mannheim, Germany), leading to two different DNA strands to produce a sense and an antisense probe for both $\beta$-actin and CD55. Both strands were translated in a digoxigenin labelled RNA probe according to the manufacturer's instructions (Boehringer), as described previously. ${ }^{20}$

\section{RNA in situ hybridisation}

RNA in situ hybridisation was performed on $5 \mu \mathrm{m}$ thick paraffin embedded sections on SuperFrost Plus slides (Menzel-Gläser, Braunsweig, Germany), as described previously. ${ }^{20}$ The following modifications were introduced: hybridisation was performed at $50^{\circ} \mathrm{C}$ for 16 hours. After hybridisation, slides were washed with $50 \%$ formamide in $2 \times \mathrm{SCC}$ at $42^{\circ} \mathrm{C}$, followed by a washing step of 45 minutes in $0.1 \times$ SSC with $20 \mathrm{mM} \quad \beta$-mercaptoethanol (Merck, Darmstadt, Germany) at $50^{\circ} \mathrm{C}$.

\section{RESULTS}

\section{mCRP expression by enteric plexuses}

Initially, normal colorectal tissue was examined for the presence of enteric plexuses. Due to the low numbers of normal colon tissue available, we expanded this group with colorectal cancer tissue to obtain more elaborate results and allow statistical analysis. To demonstrate that there was no significant difference in mCRP expression between enteric plexuses, we compared staining patterns on plexus cells of all markers between colorectal cancer sections and sections of normal colon tissues. Enteric plexuses in colorectal cancer tissue showed a similar expression pattern for MCRP as enteric plexuses in normal colon tissue $($ CD46 $\mathrm{p}=0.249$, CD55 $p=0.299$, CD59 $p=0.324$, C3d $p=0.244)$. Subsequently, colorectal cancer sections were used to analyse mCRP expression patterns in enteric plexuses.

To determine expression levels of mCRP on plexus cells, frozen tissue sections of colorectal carcinoma patients $(\mathrm{n}=35)$ were stained for CD46, CD55, and CD59 and scored for intensity of staining and percentage of positive cells. It was shown that CD46 was expressed in relatively low amounts on enteric plexus cells in contrast with CD55 and CD59 that were highly expressed. Figure l shows the cumulative scores (intensity score+score of percentage positive cells) for each marker on the colorectal cancer tissue sections.

Expression patterns of mCRP on enteric plexuses are shown in fig 2A-D (Auerbach's plexuses; submucosal plexuses showed similar staining patterns (data not shown)). High membranous expression of CD59 was observed on most cells of the enteric plexuses although some extracellular staining could not be excluded (fig 2A). Also, muscle cells stained positive for CD59. CD55 showed a membranous 

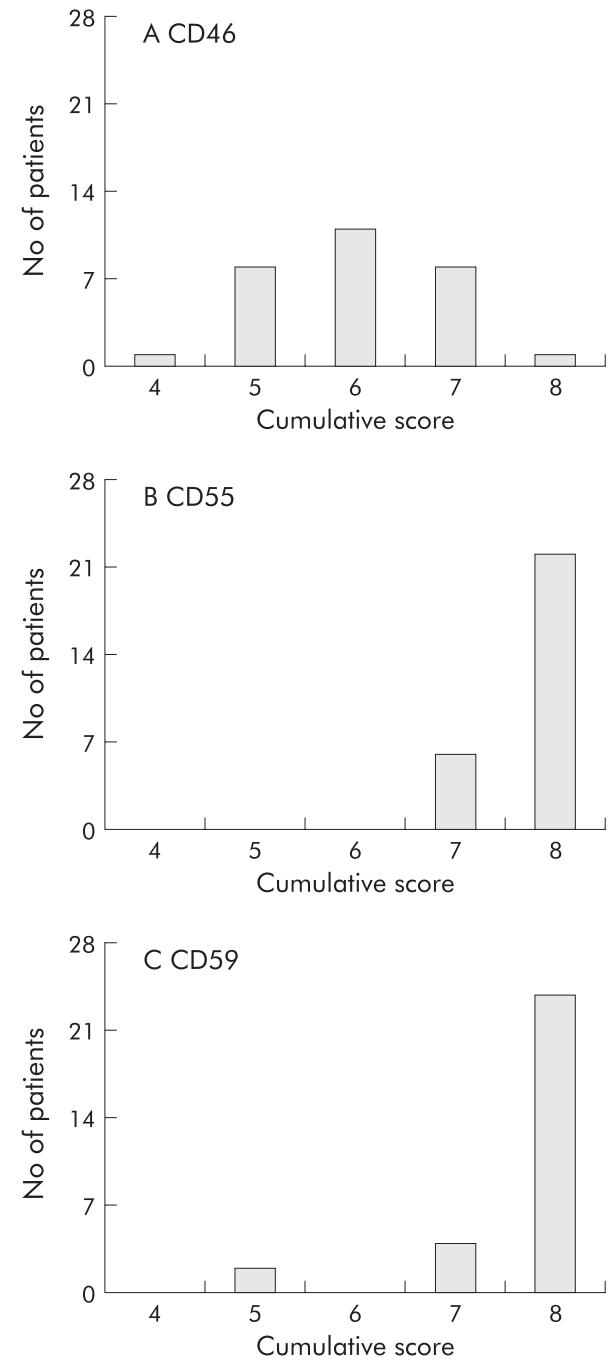

Figure 1 Expression levels of membrane bound complement regulatory proteins (mCRP) on enteric plexuses. Colorectal cancer cryostat sections $(5 \mu \mathrm{m})$ were stained using immunohistochemistry for CD46 (J4-48 (A)), CD55 (Bric216; (B)) and CD59 (Bric229 (C)) and subsequently scored for intensity of staining and percentage of positive cells in enteric plexuses, as described in the materials and methods section. The cumulative score is depicted. The number of patients with a particular score is shown. CD46 showed a weak staining pattern whereas CD55 and CD59 demonstrated high expression on cells within the enteric plexuses.

staining pattern on plexus cells but also a remarkable "ring-like" staining around the plexuses was observed that seemed not to be associated with cellular expression (fig 2B). In contrast with CD59, CD55 was not present on surrounding muscle cells. CD46 was present in low quantities in the plexus. No membranous staining pattern comparable with CD55 or CD59 staining was observed (fig 2C). Muscle cells also expressed low levels of CD46, but no CD46 staining was present in a ring around the plexuses. The observed staining pattern around the plexuses was thus unique for CD55. To exclude non-specific staining due to cross reactivity of the anti-CD55 mAb (Bric216), the expression pattern of CD55 was re-evaluated using another anti-CD55 mAb that detects a different CD55 epitope (MBC1). No differences in staining pattern between the two anti-CD55 mAb were observed (data not shown).

To study if CD55 (and CD46) expression inhibited complement deposition, the presence of $\mathrm{C} 3 \mathrm{~d}$ was investigated.
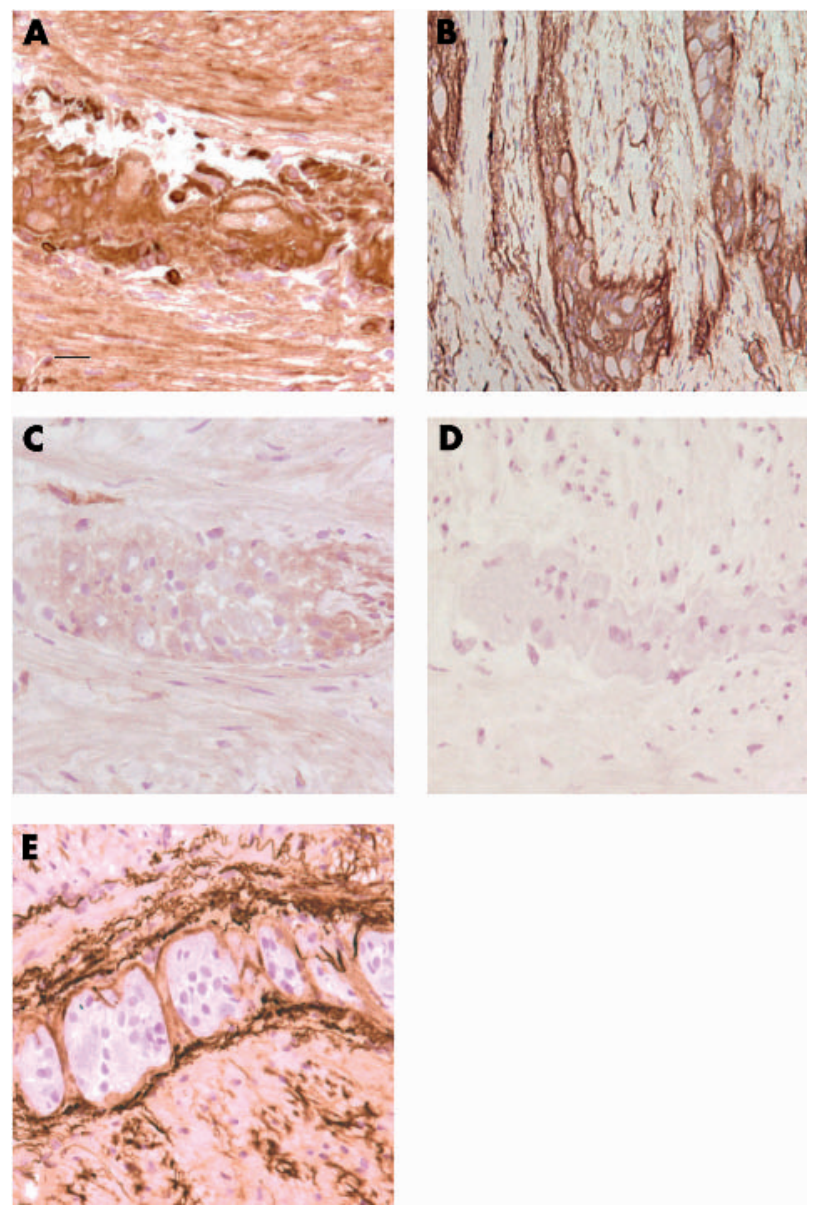

Figure 2 Expression patterns of membrane bound complement regulatory proteins (mCRP) and C3d on enteric plexuses. Colorectal cancer cryostat sections $(5 \mu \mathrm{m})$ were stained using immunohistochemistry for the presence of CD55 (Bric216), CD46 (J4-48), CD59 (Bric229), and C3d, as described in materials and methods. Auerbach's plexuses were slightly immunoreactive for CD46 (C) and highly immunoreactive for CD59 (A) and CD55 (B). In the control sections, the first monoclonal antibody was omitted; no immunoreactivity was observed (D). C3d was present around the plexus; no staining was observed on the plexus cells (E). The scale bar (in A) represents $10 \mu \mathrm{m}$ and also applies to B-E.

C3d was detected in the ring around the plexuses, co-localising with CD55 (fig 2E), whereas plexus cells themselves were negative for $\mathrm{C} 3 \mathrm{~d}$.

\section{Presence of CD55 on elastic fibres surrounding the plexuses}

To investigate whether the CD55 present around the plexuses colocalised with elastic fibres, elastin specific staining was performed on three colon carcinomas and three normal colon tissue specimens. Positive staining for elastin was found around the plexuses, at some places focally in the plexuses, and scattered through the muscle layer (fig 3A), colocalising with CD55 staining (fig 2B, fig $4 \mathrm{H}$ ). No differences in elastin staining between normal and cancer tissues were observed. Comparable staining patterns for elastin were found with Verhoef's histochemical staining that stains elastic fibres in black (data not shown), indicating colocalisation of CD55 with elastin.

To investigate if CD55 is attached to elastic fibres around plexuses, frozen sections of colon carcinoma and normal colon tissue were treated with PIPLC. CD55 staining on elastic fibres had a comparable staining intensity with 

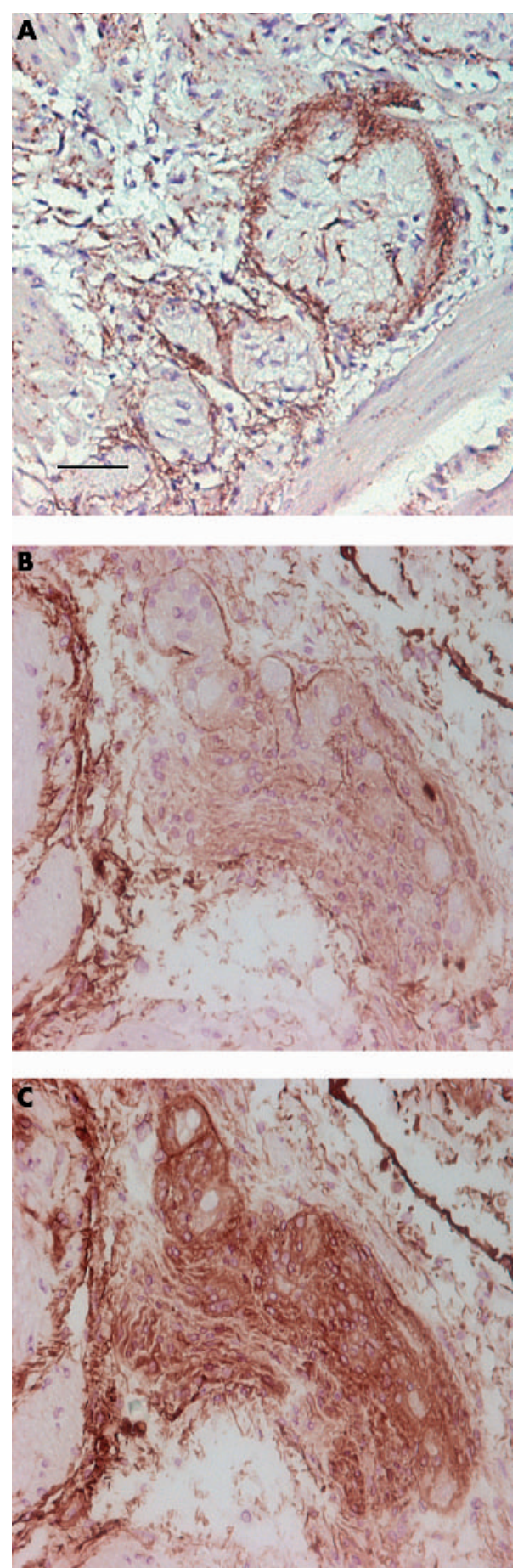

Figure 3 Elastic fibres surround Auerbach's plexuses contain CD55. Colorectal cancer cryostat sections $(5 \mu \mathrm{m})$ sections were stained using immunohistochemistry with a monoclonal antibody (mAb) directed against elastin (A) or anti-CD55 (Bric216 (B, C)), as described in material and methods. Elastic fibres were present in a band around Auerbach's plexuses. Between the muscle cells scattered immunoreactivity was observed (A). Phosphatidylinositol specific phospholipase C (PIPLC) treated submucosal plexus (B). Treatment with PIPLC cleaved CD55 from plexus cells but not from elastic fibres. The same submucosal plexus not treated with PIPLC (C). In control sections, the first mAb was omitted; no immunoreactive was observed (data not shown). The scale bar (in A) represents $25 \mu \mathrm{m}$ and also applies to B and C.
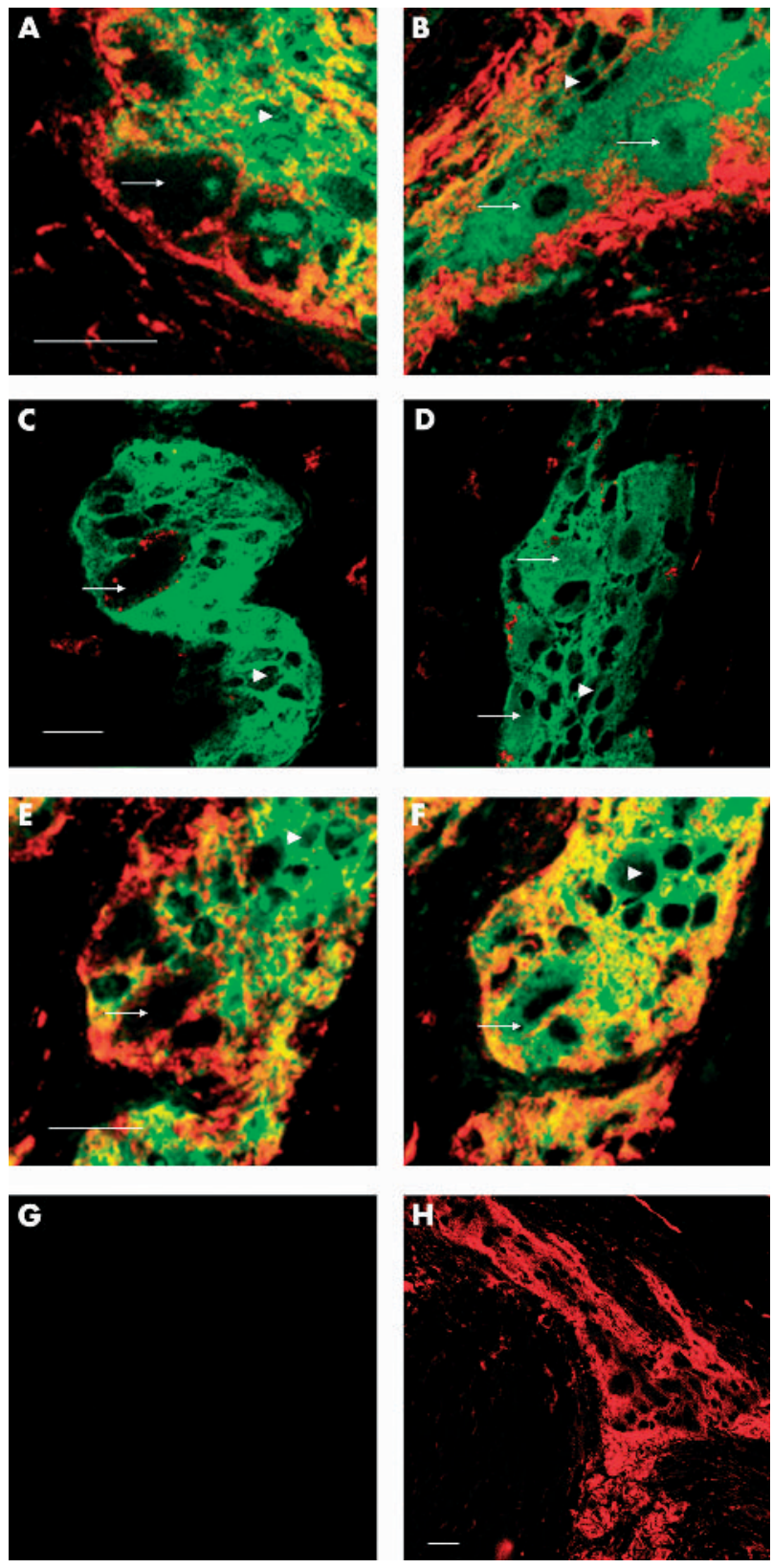

Figure 4 Neurones express CD55 and CD59. Colorectal cancer cryostat sections were double stained using immunofluorescence for CD55 (MBC1), CD46 (J4-48), or CD59 (Bric229), and S100 protein (left column) to stain enteric glial cells or protein gene product 9.5 (PGP9.5) (right column) to stain enteric neurones, respectively. Primary antibodies were detected with GaM-lgG-Alexa-546 (red; CD55, CD46, and CD59) and GaR-Ig-FITC (green; S100 and PGP9.5), respectively. CD55 immunoreactive cells, negative for $\$ 100$, are neurones (arrow; glial cell, arrowhead (A)). CD55 immunoreactive cells, PGP9.5 immunoreactive cells are neurones (arrow; glial cell, arrowhead (B)). S100 immunoreactive glial cells (arrowhead) are negative for CD46 (C). PGP9.5 immunoreactive neuronal (arrow) and glial cells (arrowhead) are negative for CD46 (D). CD59 is expressed on S100 negative neurones (arrow) and on S100 immunoreactive glial cells (arrowhead (E)). PGP9.5 immunoreactive neuronal (arrow) and glial cells (arrowhead) express CD59 (F). In control sections, the first monoclonal antibodies were omitted; no immunoreactivity was observed (G). Lower magnification, showing the ring of CD55 immunoreactivity around the plexus (CD55 staining only (H)). Pictures were obtained using a confocal microscope. The scale bars represent $10 \mu \mathrm{m}$ (both left and right panels). 
(fig 3B) or without (fig 3C) PIPLC treatment whereas CD55 staining on plexus cells was severely decreased after treatment, indicating that $\mathrm{CD} 55$ was indeed attached to elastic fibres.

\section{CD55 expression on neuronal and glial cells}

To determine the mCRP expression pattern on glial cells and neuronal cells within the plexuses, immunofluorescent double stainings were performed. Neuronal tissue was stained using the PGP9.5 antibody (green, cytoplasmic staining pattern; fig 4B, D, F). This antibody also stained glial cells. Glial cells were visualised using an anti-S100 antibody. This antibody exclusively detects glial cells (green, cytoplasmic staining pattern; fig 4A, C, E). CD55 was present in a homogeneous membranous expression pattern on neuronal cells (fig 4A, B; arrows). In addition, a strong CD55 signal was observed surrounding the plexuses and in the muscle layers (fig $4 \mathrm{H}$ ). A similar staining pattern was observed with the MBCl anti-CD55 mAb (data not shown).

In accordance with immunohistochemical staining, CD46 was detected within the plexus using immunofluorescence (fig 4C, D) although a more limited number (and only neuronal cells) of plexus cells were found to be positive. CD59 was abundantly expressed on the cell membrane of both glial and neuronal cells, as observed with both methods (fig 4E, F).

To compare mCRP expression patterns between neuronal cells and glial cells from enteric plexuses and the CNS, cryostat tissue sections of normal cortex, containing both white and gray matter, were stained for expression of CD46, CD55, and CD59, and S100 or PGP9.5, respectively (fig 5). CNS neurones were negative for CD55 (fig 5D) whereas glial cells in the CNS expressed CD55 (fig 5C). CD46 was expressed on CNS neurones (fig 5B) and was absent on the majority of CNS glial cells (fig 5A). CD59 was expressed on both CNS glia cells and on CNS neurones (fig 5E, F, respectively). No $\mathrm{C} 3 \mathrm{~d}$ could be detected in brain tissue sections (data not shown). Similar staining patterns were observed using immunohistochemistry (data not shown).

\section{Expression of CD55 mRNA by neuronal cells}

To confirm the observation that neuronal cells of the plexus express CD55, mRNA in situ hybridisation (RISH) for CD55 was performed on paraffin sections of normal and colorectal cancer tissue, as described in the materials and methods section. CD55 mRNA was indeed expressed by neuronal cells in Auerbach's (fig 6A) and submucosal plexuses (fig 6B). No CD55 mRNA was detected in enteric glial cells. CD55 mRNA expression in the villi served as an internal positive control (fig 6B). Muscle cells did not stain for CD55 mRNA (fig 6A). Endothelial cells and leucocytes, present in local lymph nodes or in the epithelial layer, were positive for CD55 mRNA (not shown). RISH for $\beta$-actin on the same tissue was used as a positive control to detect an appropriate mRNA signal. Glial cells, neurones, and muscle cells all stained positive for $\beta$-actin (data not shown). The sense probes were used as a negative control and did not result in any detectable signal (data not shown).

\section{Expression of CD55 on ganglioneuroma tissue}

To investigate if the CD55 expression level can be altered on plexus neurones when normal plexus morphology is disturbed, the expression level of CD55 mRNA was determined on appendix ganglioneuroma tissue using RISH. In this tumour tissue consisting of malignant neuronal cells and stroma, no distinct plexuses were recognised (fig 6C, D). CD55 mRNA was overexpressed on these cells compared with normal plexus neurones (fig 6D).
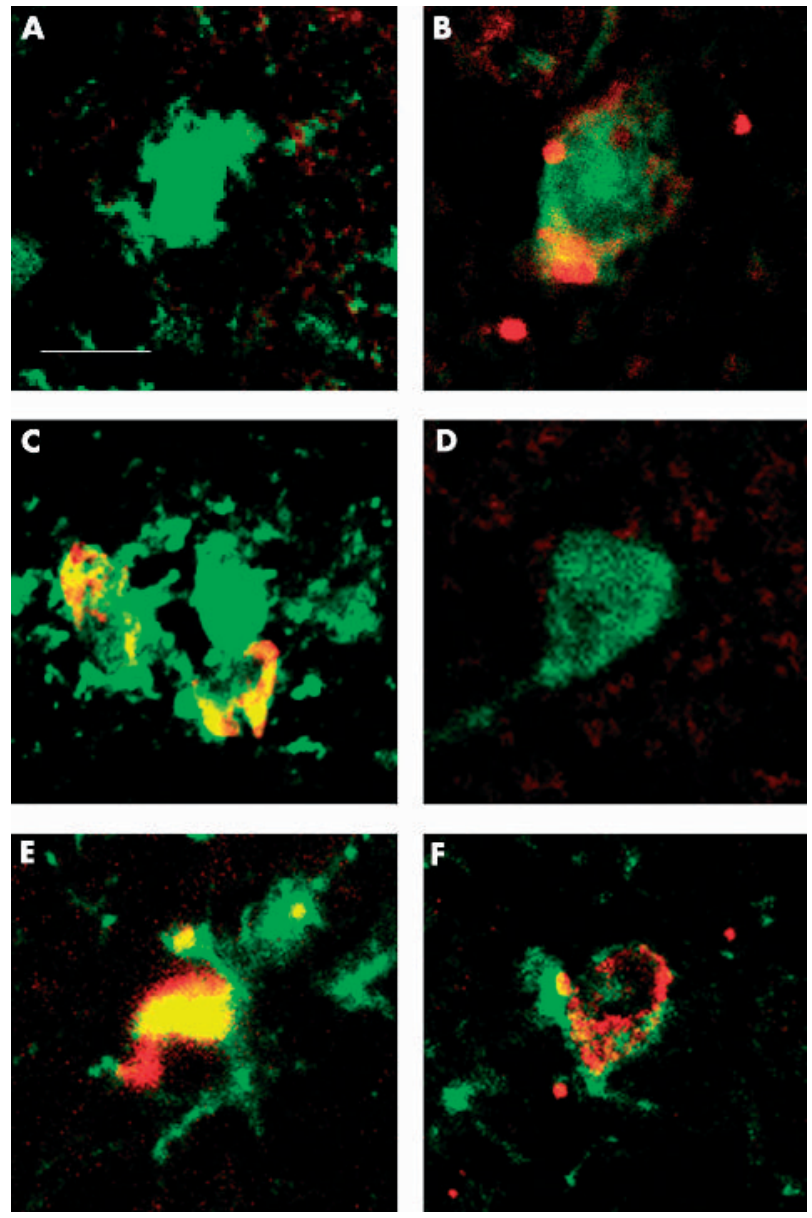

Figure 5 Expression patterns of membrane bound complement regulatory proteins (mCRP) in the central nervous system. Brain cryostat sections were double stained using immunofluorescence for CD46 (J4$48)$, CD55 (MBC1), or CD59 (Bric229), and S100 protein (left column) to stain glial cells or protein gene product 9.5 (PGP9.5) (right column) to stain neurones, respectively. Primary antibodies were detected with GaM-lgG-Alexa-546 (red; CD55, CD46, and CD59) and GaR-lg-FITC (green; S100 and PGP9.5). CD46 was expressed in very low amounts on occasional glial cells (A). CD46 was expressed in low amounts on neurones (B). Glial cells expressed CD55 in high levels (C). Neurones were negative for CD55 (D). CD59 was expressed on glial cells (E). CD59 was expressed on neurones at intermediate levels (data not shown). The scale bar (in A) represents $10 \mu \mathrm{m}$ and also applies to B-F.

\section{DISCUSSION}

In the gut, as a port of entry for antigens and pathogens, complement mediated damage of neuronal tissue is likely to occur when insufficient amounts of complement regulatory proteins, such as CD46 and CD55, are expressed. In the present study, mCRP expression patterns on plexus cells were investigated. It was shown that enteric neurones expressed CD55 in contrast with CNS neurones. In accordance with previous studies, CNS neurones were shown to express minimal amounts of CD46 and CD59 and lack CD55. ${ }^{16} 182122$ An explanation for the difference in CD55 expression may be that CNS neurones are generally not exposed to antigens, pathogens, and activated complement components due to the presence of the blood-brain barrier whereas neurones of the enteric plexuses might be exposed more often to an inflammatory process. ${ }^{1}$ Therefore, it is conceivable that enteric neurones have to upregulate CD55 expression to protect themselves from complement mediated injury. ${ }^{23}$

In contrast with neuronal cells, glial cells in the CNS have been described to express CD46, CD55, and CD59. ${ }^{22-27}$ In the 

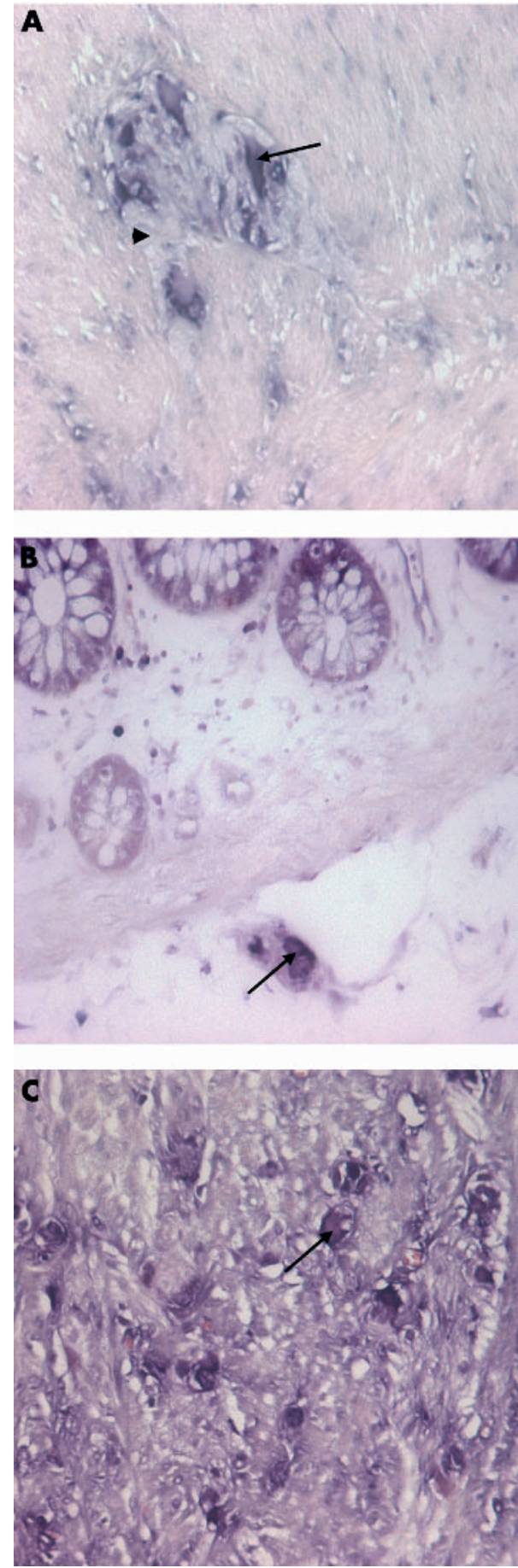

Figure 6 Enteric neurones express CD55 mRNA. RNA in situ hybridisation (RISH) was performed on colorectal cancer tissue and normal colon tissue sections, as described in material and methods. Auerbach's (A) and submucosal (B) plexus neurones (arrow) express CD55 mRNA in contrast with the surrounding glial (arrowhead) and muscle cells. No differences in mRNA expression in enteric neurones were observed between normal colon and cancer tissue. RISH revealed high expression levels of CD55 mRNA in ganglioneuroma cells (C) (arrow). The sense probe was used as a control and did not result in any signal (data not shown).

present study, no CD55 RNA could be demonstrated in enteric glial cells whereas protein expression was observed in CNS glial cells. This suggests that enteric glial cells are protected against complement mediated injury in a different way.
Acellular CD55 staining observed around the plexuses suggests that this CD55 might provide an alternative protection mechanism for complement mediated injury of CD55 negative glial cells. It has previously been observed that CD55 can be covalently attached to elastic fibres, ${ }^{28}{ }^{29}$ and that elastic fibres are associated with enteric plexuses. ${ }^{30} \mathrm{CD} 55$ has been shown to bind to elastic fibres when it is in the phosphoinositol (PI) anchorless form. ${ }^{28-31}$ We demonstrated here that PIPLC treatment cleaved CD55 from cells within the plexuses but not from the surrounding elastic fibres, ${ }^{32}$ suggesting that it is indeed covalently linked to these fibres. This CD55 is most likely produced by enteric neurones because these are the only cells in the vicinity of the plexuses positive for both CD55 mRNA and protein.

To investigate the efficiency of CD55 expression on neurones and elastic fibres with regard to complement activation, the deposition pattern of C3d was determined in and around the enteric plexuses. C3d was chosen because this split product of C3 remains attached to the target cell membrane after degradation by factor I. It was observed that both plexus neurones and glial cells were negative for C $3 \mathrm{~d}$. In contrast, the ring of elastic fibres that stained positive for CD55 was also highly positive for C3d. This indicates that elastic fibre bound CD55 is indeed very effective in controlling complement activation, resulting in split product deposition near the elastic fibre bound CD55, and thus protecting the cells of the enteric plexus.

Abnormalities in mCRP expression on enteric neurones and glial cells might affect the course of diseases affecting peripheral neuronal tissue. Previously, associations have been made between plexus abnormalities and inflammatory conditions $^{5}$; for example, in Crohn's disease, where disruption of the enteric glial network is an early event in pathogenesis $^{58}$ or in Hirschprung's disease where during development neurones fail to migrate to the intestine. ${ }^{8}$ On complement activation, by for example autoantibodies ${ }^{5}$ or bacteria, CD55 can provide the essential protection of enteric plexuses against induction of an inflammatory response, mediated via complement fragments such as $\mathrm{C} 5 \mathrm{a}$ or iC $3 \mathrm{~b}$ or direct complement mediated lysis. Downregulation of mCRP might allow complement mediated injury and result in onset or progression of disease.

In contrast, upregulation of mCRP expression may offer protection against complement mediated attack. Overexpression of mCRP has been described for several tumour types (for example, colorectal cancer). ${ }^{11} 3334$ Overexpression of CD55 by tumours originating from neuronal tissue is however not usual. Neuroblastoma cells do not or minimally express this mCRP. ${ }^{32}$ In the present study, we have demonstrated that ganglioneuroma cells derived from intestinal tissue overexpress CD55 mRNA compared with normal enteric neurones. This suggests that CD55 expression can be modulated on enteric neuronal cells. These altered CD55 expression levels may affect the course of disease.

In summary, the present study describes for the first time CD55 mRNA expression in peripheral neurones and lack of CD55 mRNA expression of peripheral glial cells. Furthermore, our study suggests that CD55 negative peripheral glial cells in the colon may effectively be protected by CD55 attached to elastic fibres that surround the enteric plexuses. Finally, we have demonstrated that, in contrast with tumours originating from other neuronal tissue, CD55 expression is increased on neuroganglioma cells, suggesting that the level of mCRP expression on enteric neurones may affect the course of disease.

\section{ACKNOWLEDGEMENTS}

We thank pathologist Dr J Morreau and neuropathologist Dr SG van Duinen for their assistance in histology and J Broderick Smith for improving the English. This study was sponsored by the Dutch Cancer Society, grant No 99032. 


\section{Authors' affiliations}

K A Gelderman, H J M A A Zijlmans, M J Vonk, A Gorter, Department of Pathology, Leiden University Medical Centre, Leiden, the Netherlands

\section{REFERENCES}

1 Sharkey KA, Kroese AB. Consequences of intestinal inflammation on the enteric nervous system: neuronal activation induced by inflammatory mediators. Anat $\operatorname{Rec} 2001 ; 262: 79-90$.

2 Wood JD, Alpers DH, Andrews PL. Fundamentals of neurogastroenterology. Gut 1999:45(suppl 2):116-16.

3 Timmermans JP, Hens J, Adriaensen D. Outer submucous plexus: an intrinsic nerve network involved in both secretory and motility processes in the intestine of large mammals and humans. Anat Rec 2001;262:71-8.

4 Gershon MD, Rothman TP. Enteric glia. Glia 1991;4:195-204.

5 Cornet A, Savidge TC, Cabarrocas J, et al. Enterocolitis induced by autoimmune targeting of enteric glial cells: a possible mechanism in Crohn's disease? Proc Natl Acad Sci U S A 2001;98:13306-11.

6 Collins SM. The immunomodulation of enteric neuromuscular function: implications for motility and inflammatory disorders. Gastroenterology 1996;111:1683-99.

7 MacDonald $\pi$. Depletion of the enteric nervous system by gene targeting results in haemorrhagic necrosis of the ileum. Gut 1998;43:742-3.

8 Teixeira MM, Gazzinelli RT, Silva JS. Chemokines, inflammation and Trypanosoma cruzi infection. Trends Parasitol 2002;18:262-5

9 Zimmermann-Nielsen E, Baatrup G, Thorlacius-Ussing O, et al. Complement activation mediated by mannan-binding lectin in plasma from healthy individuals and from patients with SLE, Crohn's disease and colorectal cancer. Suppressed activation by SLE plasma. Scand J Immunol 2002;55:105-10.

10 Laufer J, Oren R, Goldberg I, et al. Cellular localization of complement C3 and C4 transcripts in intestinal specimens from patients with Crohn's disease. Clin Exp Immunol 2000;120:30-7.

11 Gorter A, Meri S. Immune evasion of tumor cells using membrane-bound complement regulatory proteins. Immunol Today 1999;20:576-82.

12 Nicholson-Weller A, Wang C. Structure and function of decay accelerating factor CD55. J Lab Clin Med 1994; 123:485-91.

13 Seya T, Turner JR, Atkinson JP. Purification and characterization of a membrane protein (gp45-70) that is a cofactor for cleavage of $\mathrm{C} 3 \mathrm{~b}$ and $\mathrm{C} 4 \mathrm{~b}$. J Exp Med 1986;163:837-55.

14 Meri S, Morgan BP, Davies A, et al. Human protectin (CD59), an 18,000$20,000 \mathrm{MW}$ complement lysis restricting factor, inhibits C5b-8 catalysed insertion of C9 into lipid bilayers. Immunology 1990;71:1-9.

15 Singhrao SK, Neal JW, Rushmere NK, et al. Differential expression of individual complement regulators in the brain and choroid plexus. Lab Invest 1999;79:1247-59.

16 Asghar SS. Membrane regulators of complement activation and their aberrant expression in disease. Lab Invest 1995:72:254-71.

17 Singhrao SK, Neal JW, Rushmere NK, et al. Spontaneous classical pathway activation and deficiency of membrane regulators render human neurons susceptible to complement lysis. Am J Pathol 2000;157:905-18.
18 Gelderman KA, Blok VT, Fleuren GJ, et al. The inhibitory effect of CD46, CD55, and CD59 on complement activation after immunotherapeutic treatment of cervical carcinoma cells with monoclonal antibodies or bispecific monoclonal antibodies. Lab Invest 2002:82:483-93.

19 Ruiter DJ, Ferrier CM, Van Muijen GNP, et al. Quality control of immunohistochemical evaluation of tumour-associated plasminogen activators and related components. Eur J Cancer 1998;34:1334-40.

20 De Boer WI, Sont JK, van Schadewijk A, et al. Monocyte chemoattractant protein 1, interleukin 8, and chronic airways inflammation in COPD. J Pathol 2000;190:619-26

21 Gasque $\mathbf{P}$, Thomas $A$, Fontaine $M$, et al. Complement activation on human neuroblastoma cell lines in vitro: Route of activation and expression of functional complement regulatory proteins. J Neuroimmunol 1996;66:29-40.

22 Morgan BP, Gasque P. Expression of complement in the brain: Role in health and disease. Immunol Today 1996;17:461-6.

23 Koski CL, Estep AE, Sawant-Mane S, et al. Complement regulatory molecules on human myelin and glial cells: Differential expression affects the deposition of activated complement proteins. J Neurochem 1996;66:303-12.

24 Yang C, Jones JL, Barnum SR. Expression of decay-accelerating factor (CD55), membrane cofactor protein (CD46) and CD59 in the human astroglioma cell line, D54-MG, and primary rat astrocytes. J Neuroimmunol 1993;47:123-32.

25 Gasque P, Morgan BP. Complement regulatory protein expression by a human oligodendrocyte cell line: cytokine regulation and comparison with astrocytes. Immunology 1996;89:338-47.

26 Scolding NJ, Morgan BP, Compston DAS. The expression of complement regulatory proteins by adult human oligodendrocytes. J Neuroimmunol 1998; 84:69-75

27 Chen SH, Caragine T, Cheung NKV, et al. Surface antigen expression and complement susceptibility of differentiated neuroblastoma clones. Am J Pathol 2000:156:1085-91.

28 Werth VP, Ivanov IE, Nussenzweig V. Decay-accelerating factor in human skin is associated with elastic fibers. J Invest Dermatol 1988;91:511-16.

29 Werth VP, Shi X, Kalathil E, et al. Elastic fiber-associated proteins of skin in development and photoaging. Photochem Photobiol 1996;63:308-13.

30 Liberti EA, Gaspar LP, de Carvalho CA, et al. A morpho-quantitative study of the myenteric ganglia throughout the human digestive tract. Rev Hosp Clin Fac Med Sao Paulo 1998;53:55-60.

31 Sayama K, Shiraishi S, Shirakata Y, et al. Characterization of decayaccelerating factor (DAF) in human skin. J Invest Dermatol 1991;96:61-4.

32 Yang LB, Li R, Meri S, et al. Deficiency of complement defense protein CD59 may contribute to neurodegeneration in Alzheimer's disease. J Neurosci 2000:20:7505-9.

33 Schmitt CA, Schwaeble W, Wittig BM, et al. Expression and regulation by interferon-gamma of the membrane-bound complement regulators CD46 (MCP), CD55 (DAF) and CD59 in gastrointestinal tumours. Eur J Cancer [A] 1999:35:117-124.

34 Li L, Spendlove I, Morgan J, et al. CD55 is over-expressed in the tumour environment. Br J Cancer 2001;84:80-6. 